\title{
Feasibility Study of Interactive Game Technologies to Improve Experience with Inhaler Spacer Devices in Young Children
}

\author{
Tariq M. Aslam · Savana Shakir · Clare Murray
}

Received: October 19, 2016 / Published online: December 8, 2016

(C) The Author(s) 2016. This article is published with open access at Springerlink.com

\begin{abstract}
Introduction: The correct use of inhaler devices with facemasks and spacers in young children can be difficult for both children and parents, resulting in distress for both, poor adherence and ineffective drug delivery. The aim of this study was to develop and assess the utility and impact of an interactive electronic game designed to improve the experience of spacer devices in young asthmatic children.
\end{abstract}

Enhanced content To view enhanced content for this article go to http://www.medengine.com/Redeem/ 8327F0601B0122CC.

T. M. Aslam

Division of Pharmacy and Optometry, Faculty of Biology, Medicine and Health, University of

Manchester, Manchester Academic Health Science

Centre, Central Manchester University Hospitals

NHS Foundation Trust, Manchester, UK

S. Shakir - C. Murray

Central Manchester University Hospitals NHS

Foundation Trust, Manchester, UK

C. Murray ( $\square)$

Division of Infection, Immunity and Respiratory Medicine, Faculty of Biology, Medicine and Health, University of Manchester, Manchester Academic Health Science Centre, University Hospital of South Manchester NHS Foundation Trust, Manchester, UK e-mail: Clare.murray@manchester.ac.uk
Methods: The Respiratory Aid For Inhaler (RAFIhaler) technology consists of a smartphone mounted on a metered dose inhaler with a valved holding chamber device (spacer) and connected to an electronic sensor adjacent to the outflow valve of the spacer. A custom-designed android application on the smartphone derives the child's breathing patterns from the sensor, translating this information into visible effects on on-screen cartoon characters. After a period of iterative development, the final structure and software design was tested on children at Royal Manchester Children's Hospital who had been admitted with acute wheeze. A survey, including open-ended questions, was administered to assess the parent and child's reaction to the RAFIhaler.

Results: A total of 14 children (age range 2-7 years; 7 boys) participated in the study and used the RAFIhaler, of whom 13 completed the final survey; all 14 parents completed the survey. All 13 children reported they enjoyed playing the game. Ten of these children (91\%) felt that the RAFIhaler helped them in taking their medication, and 13 of the 14 parents felt that RAFIhaler helped their 
children use their spacer. The one parent who felt that the RAFIhaler was not of any benefit felt his/her child already used the inhaler well. Some of the benefits of the RAFIhaler specifically voiced by parents were "enjoyable" $(n=3)$, "good distraction" $(n=3)$, "made child calmer" $(n=2)$, "helped in breathing/ inhalation technique" $(n=3)$ and "would be useful in home setting" $(n=1)$.

Conclusion: Young children may benefit from the incorporation of devices and game applications with use of their inhalers. Feedback from children and parents suggests potential benefits to children may include learning correct use, reducing distress and anxiety and improving overall adherence.

Keywords: Android application; Asthma; Children; Device; Inhaler; Spacer; RAFIhaler

\section{INTRODUCTION}

Asthma is the commonest chronic disease of childhood and a leading cause of childhood morbidity [1]. Children may also present acutely with wheeze from other causes, such as viral infections. Studies in the UK show around a $25 \%$ prevalence of wheeze from all causes in children over a 3 year period [2]. Other European studies show that approximately one-third of all children before the age of 8 years will have required some form of inhalation therapy for wheeze [3].

Metered dose inhalers (MDI) used with spacer devices are invaluable to allow children to gain the benefit of inhaled treatments in an effective way that can be used anywhere, without the need of nebulisers to deliver the treatments. Studies have shown that these devices, when used correctly, can match the efficacy of nebulisers for the treatment of asthmatic children [4]. Many children are also given regular inhaled preventer medication to take together with reliever inhaler medication. There is good evidence that adherence with inhalers in young children is often poor, with studies using electronic logging devices showing mean adherence rates of between 30 and 70\% [5]. Inhaled treatment has its own special challenges. The treatment itself is time-consuming, especially in pre-school children. Some young children find the experience of a spacer with a close fitting face-mask frightening, which can add to the challenges faced by parents trying to administer asthma medications regularly. A good seal with the facemask is required-a poor seal greatly reduces drug delivery [6]. Drug deposition is also significantly reduced if the child is crying or screaming rather than breathing normally [6].

Asthma is a disease which is often characterised by long spells of good health interspersed by episodes of breathlessness and wheeze. For those on preventer treatments it is necessary that these treatments continue to be given throughout periods of well-being to prevent symptomatic episodes; young children may need incentives to encourage this. Those not on regular preventers only use their inhalers when unwell, and parents may find their child less co-operative at such times, just when they need the benefit of their inhaler most.

The aim of this feasibility study was to develop an electronic game device using a smartphone to encourage correct use of a valved holding chamber and facemask. We assessed the response to the device from both patients and their caregivers in an acute setting.

\section{METHODS}

The Respiratory Aid For Inhalers (RAFIhaler) was developed through a period of iterative testing, design and programming. The final version 


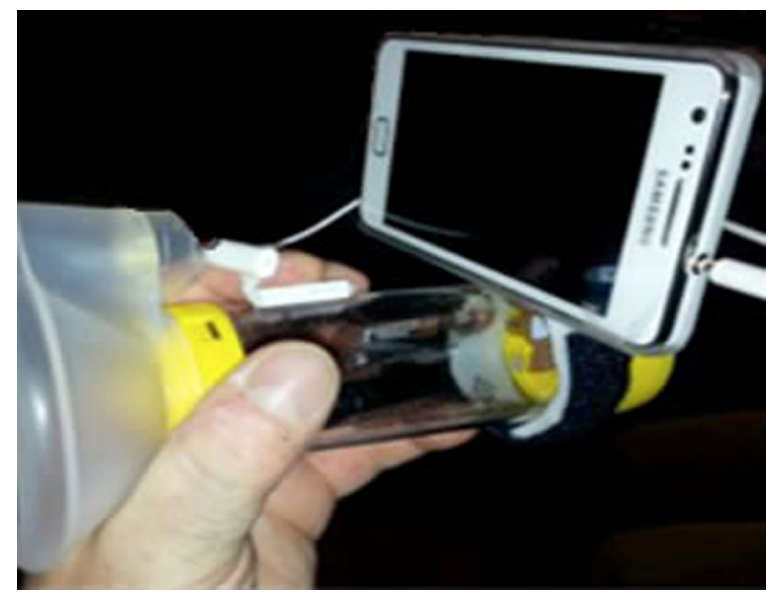

Fig. 1 Respiratory Aid For Inhalers (RAFIhaler) mounted on a paediatric aerochamber

assessed in this study comprises an android application installed on a smartphone, which is itself mounted on top of a valved holding chamber device (Fig. 1). An electronic microphone sensor detects acoustic signals adjacent to the outflow valve of the spacer and provides input to the custom-designed android application on the smartphone. The sensor is placed outside of the spacer and does not come into direct contact with the valve or mask to ensure there is no danger of aspiration. The application monitors the acoustic input from the sensor every tenth of a second and algorithms derive information on the child's breathing pattern. The installed software thereby detects the correct use of the inhaler and spacer and rewards this behavior by effecting responses from the cartoon graphics on the screen (Figs. 2, 3). For example, correct breathing with a good mask seal leads to the appearance on the screen of unfriendly characters being blown away, fires being blown out, etc. with the goal to help the lead character (RAFI) escape the cartoon foes. Each game consists of five scenes which can be completed with five good breaths.

The initial prototype was developed by a father (TA) for his son (Rafi) who was very difficult to manage acutely because of

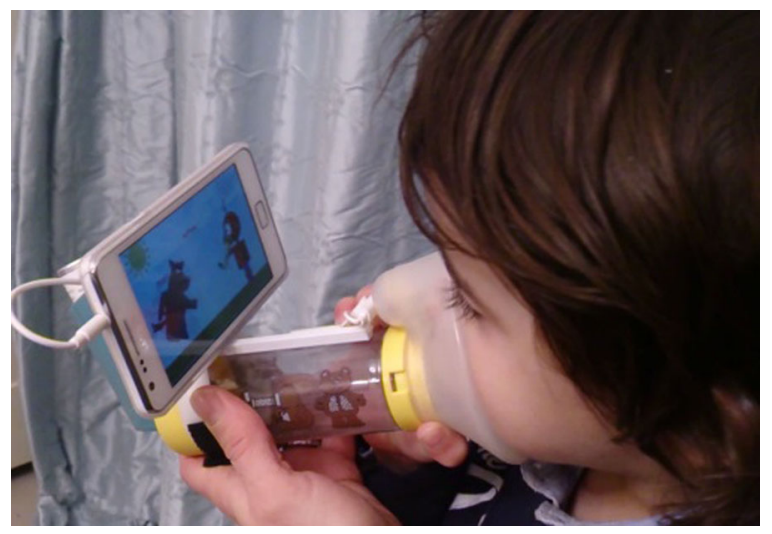

Fig. 2 RAFIhaler in use

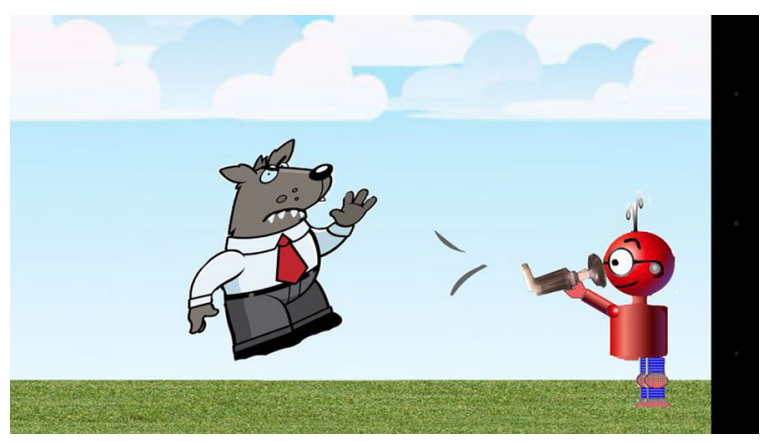

Fig. 3 Example cartoon: 'bad wolf is blown away by proper breathing

intolerance to his prescribed spacer. Although the final device could be useful in chronic care, the current feasibility study was designed to reflect this original acute setting. The final RAFIhaler was therefore tested on 14 children (age range 2-7 years) at Royal Manchester Children's Hospital who had been admitted with acute wheeze. The study had local ethical approval, and all procedures followed were in accordance with the ethical standards of the responsible committee on human experimentation (institutional and national) and with the Helsinki Declaration of 1964, as revised in 2013. Caregivers gave written informed consent, and children gave assent to the main study and also separately to themselves being videoed for further assessment. Instructions on the correct breathing technique 
and use of the spacer were given by the child's medical team. Children and parents were recruited by an independent researcher not involved in the device creation or direct patient care. She approached the children and their caregivers and explained the purposes of the study and explained to the children that whilst they were using their inhalers they would be able to play a game which worked as long as they used their inhaler correctly, as previously directed by their healthcare professional. She explained that when they breathed correctly they would see things happen on the screen and explained, with some examples, the different games involved. After each child used the RAFIhaler, a survey was administered to assess whether the children enjoyed taking their inhaler/playing and whether the parent or child perceived a benefit from the RAFIhaler in helping them use their inhaler. Any further feedback volunteered was also recorded. The precise questions asked are detailed below.

Questions to child:

1. Did you like playing?

2. What did you like about it?

3. What did not you like about it?

4. What would make it better?

5. Did it help you have the medicine?

Questions to caregiver:

1. Do you think this helped your child have the medicine?

2. What were the good and bad points?

3. How could it be improved?

In addition, following use of RAFIhaler, all patients' nurses were asked if they had any comments to make.

\section{RESULTS}

Fourteen children ( 7 girls, 7 boys) between the ages of 2 and 7 years (median age 5 years, interquartile range 2 years) participated in the study. Thirteen children and all 14 parents completed the survey. Not all individual questions were answered, possibly because the children may have been unwilling/too shy or distracted to respond or merely had no specific comment to make.

All children stated they enjoyed playing the game. Eleven children responded to further questioning, of whom ten (91\%) felt that the RAFIhaler helped them in taking medication. One child (aged 5 years) felt that he was already good at using his inhaler but still liked playing the game.

When parents were asked if they felt that RAFIhaler helped their children use their spacer, all but one thought that it did. Interestingly, of the 13 parents who felt that the RAFIhaler helped their child in taking medication, four believed their child already had a good inhaler technique, and only three felt their child really struggled to use the inhaler.

The study used a prototype which clearly had some technical issues; the microphone displaced on occasion, the smartphone slipped a little in its support and the software needed restarting on one occasion. It was also evident that the software would require some adaptation to prevent children breathing too quickly or too hard, particularly if they became excited.

The full results of the RAFIhaler survey are presented in the following subsections.

\section{Questions To Child (Identifier Followed by Age in Years)}

1. Did you like playing?

- $13 / 13$ respondents said yes

2. What did you like about it?

- 11 responses, all 11 expressing enjoyment of games and characters

A, 5: I liked the robot. It was fun. 
B, 6: Animation and cartoon characters enjoyed playing different levels.

C, 4: Cartoons, sharks (blowing sharks away), playing games and blowing out fires

D, 7: Boat game, wind/footballs

E, 4: Like the characters and playing on

F, 4: It was fun computers

G, 5: Liked cartoon-good game

$\mathrm{H}, 5$ : Different games/levels/all the games

I, 2: Looking at computer screen

$\mathrm{K}, 6$ : Good, Blowing and aiming

L, 5: Cartoon games.

3. What did you not like about it?

A, 5: I did not like the sharks

B, 6: Should be easier to play to get into different levels-it stopped working and needed to be

C, 4: Nothing restarted by moving microphone

D, 7: No

E, 4: No

F, 4: Nothing

G, 5: No

$\mathrm{K}, 6$ : Did not like the boat (it was going backwards)

L, 5: Repeated so could get bored.

4. What could make it better?
A, 5: It was fine
$\mathrm{D}, 7$ : More levels more baddies
G, 5: Nothing
K, 6: Yes
L, 5: More games.

5. Did it help you have the medicine? (adult comments in parentheses)
A, 5: Yes
B, 6: Yes
C, 4: Yes
D, 7: Yes
E, 4: Yes
$\mathrm{F}, 4$ : Yes
G, 5: No (normally OK at taking inhalers)
H, 5: Yes

I, 2: Yes (but takes inhaler well, hadn't used inhaler before admission yesterday

K, 6: Yes but good compliance)

L, 5: Yes (frightened of inhaler at first but seemed to accept inhaler with ease and take good deep breaths with games).

\section{Questions To Caregiver}

1. Do you think this helped your child have the medicine?

- 13/14 caregivers said yes. 1/14 said "no" but expressed it was a "good idea"

A, 5: Yes

B, 6: Yes. Good distraction and helped breathing

C, 4: Yes, She really enjoyed taking the inhaler and playing the games and wanted to play again. But she does normally take inhalers at home and already good with technique

D, 7: Yes, but normally good at taking inhaler-has been doing so for 2 years

E, 4: Yes

F, 4: Yes

G, 5: No but good idea

J, 6 Yes, J came into room crying, usually very difficult to get inhaler into him. Drawn in by cartoon. He became calmer and allowed the mask to be held in place

K, 6: Yes, made it more enjoyable

L, 5: He doesn't normally take inhalers but came in last night and required frequent inhalers. Taken to ward for hourly inhalers and seemed unsettled and frightened at 
taking the medication. The RAFIhaler helped ease his concerns, acted as a distraction whilst breathing in the medication. Improved inhaler technique with bigger inhalation and expiration was apparent

M, 5: Yes, it has definitely helped in breathing technique. Ted was previously taking shallow breaths but with the game managed to focus on deeper breaths and concentrated on playing the game

N, 6: It did distract her. I think it would be useful but at home setting.

2. What were the good and bad points?

A, 5: It was simple and easy for my child and kept him interested

B, 6: Overall good-slight problem with needing to move microphone

C, 4: Had to rearrange game to adjust microphone. Made her breathe quicker - Had to tell her to breathe slowly and deeply

D, 7: Good distraction on phone

E, 4: Good distraction-helps him to concentrate. Negative-blows too hard and faster than normal

F, 4: -

G, 5: Good distraction from taking inhaler

$H, 5$ : Overall good impression of RAFIhaler game

J, 6: Likes pictures

K, 6: Good idea

L, 5: Good distraction and improved technique. New to inhalers so made him less scared

M, 5: positive-helps to direct the child's attention and focus on breathing. Enjoyable activity. No bad points-a really good idea. A good way to introduce inhalers.
3. How could it be improved?

- 11 comments: 7 expression satisfaction with current game. 2 criticising that child should be advised to breathe slowly, 1 criticising microphone arrangement.

A, 5: I was happy with this just as it was

C, 4: Help with slowing down breathing but good idea and useful for entertaining children

D, 7: Microphone and blowing hard

E, 4: Instructions to breath slowly

G, 5: No, good as it is

$\mathrm{H}$, 5: Good as it is

I, 2: More levels

$\mathrm{K}, 6$ : J is normally good at taking inhaler but helped him breathe harder

L, 5: More games, easier to handle, child moving around quite a bitslightly distracted by video recording him playing RAFIhaler needed rearranging to ensure microphone and computer remained in position M, 5: Candle game to blow out candles.

Comments spontaneously voiced by parents about the device: "enjoyable" $(n=3)$; "good distraction" $(n=3)$; "made child calmer" $(n=2)$; "helped in breathing/inhalation technique" $(n=3)$; "would be useful at home setting" $(n=1)$.

Quotes from parents who took part in the study:

"He came into the room crying, usually very difficult to get inhaler into him. He was drawn in by the cartoon. He became calmer and allowed the mask to be held in place" (J, aged 2 years).

“He doesn't normally take inhalers but came in last night and required frequent inhalers. He was having hourly inhalers and seemed unsettled and frightened at taking the 
medication. The 'RAFIhaler' helped ease his concerns, acted as a distraction whilst breathing in the medication. Improved inhaler technique with bigger inhalation and expiration was apparent" (L, aged 5 years) "Yes, it has definitely helped in breathing technique. $\mathrm{T}$ was previously taking shallow breaths but with the game managed to focus on deeper breaths and concentrated on playing the game" (M, aged 2 years)

\section{Nurses' Comments}

There were comments made by three respiratory nurses who observed the study:

Nurse 1: Very good idea. Had to advise the patient to breathe slowly at times as they wanted to reach the next level

Nurse 2: Really good idea. Easy to handle and children may be very responsive to cartoon

Nurse 3: Great idea. Easy to use and good distraction for children-makes inhaler delivery so much easier.

\section{DISCUSSION}

The prototype RAFIhaler was developed and then tested on 14 children with wheeze in an acute paediatric ward. The study was semi-qualitative and used open questions, and we acknowledge the limitation of the observational study design with the absence of controls and limited numbers of participants. However, all children appeared to accept the inhaler/spacer and face mask with the RAFIhaler in place. All children who responded (13/14) stated they enjoyed using the RAFIhaler, and 13/14 caregivers felt it helped their children accept having their medicine. Overall, positive comments far outweighed negative ones, and the device appeared to offer benefit in a variety of ways. For children new to inhalers, it particularly appeared to diffuse tension and apprehension and distract from any discomfort from the mask application. Other more experienced children were seen and noted to have improved mask seals as loose masks would have caused less efficient game play. Finally, enjoyment and amusement at the cartoon characters meant that all children enjoyed the process of inhaler use. Most children who participated needed to take ten puffs of the reliever medication as they were acutely wheezy, which was quite a time-consuming process. This prototype version had a limited number of game cycles, and two children (age 5 and 7 years, respectively) mentioned the need for more games, otherwise they may easily become bored with it. This limited number of game cycles is clearly an aspect of the RAFIhaler which requires addressing in future iterations.

For this feasibility study we chose only to assess the acceptability of the device by children and their caregivers, as well as the opinions of these two groups of the device using both closed and open ended questions. Further studies will be needed, with controls, to prove more focussed outcomes, such as levels of bronchodilation and drug deposition. In this study we did not attempt to assess such outcomes and are as yet unable to verify whether they are affected by the use of the RAFIhaler. We also tested the use of RAFIhaler in the acute setting. Children only used the device on one occasion, and we have no information as to how useful such a device would be in the longer term. It may be that the use of the RAFIhaler helps some children learn to accept masks while for others continued access to the device may be required. A simple 
incentive device consisting of a fixed toy attached to the side of the spacer (Funhaler ${ }^{\mathrm{TM}}$; Alliance Tech Medical Inc., Granbury, TX) has previously been shown to improve adherence over a 2-week period but not over 3 months $[7,8]$. This finding reiterates the need for variety in the incentive device and the need to provide a number of games/levels, as pointed out by some of the older children in our study. A further study using the Funhaler ${ }^{\mathrm{TM}}$ did demonstrate improved spacer technique, indicating a potential benefit for such incentive devices [9].

\section{CONCLUSION}

The results of this small preliminary study highlight a positive attitude towards the use of gaming and interactive technologies in both young children and their caregivers in the context of the need for inhaled medication. Our findings demonstrate that such devices may be of use in encouraging young children to correctly use their inhaler with a facemask and spacer. Improvements and adaptations will need to be made to the device described here based upon the feedback obtained, with the ultimate aim of producing improved devices and applications to improve the technique of use with a paediatric spacer and adherence to treatment. Further studies should assess the potential benefits in terms of improving technique and ultimately in reducing chances of acute exacerbations through maintained adherence and disease control.

\section{ACKNOWLEDGEMENTS}

This study was supported by a Grant from CMFT Research and Innovation Division Joint Awards Scheme, funded by RMCH Charity.
Compliance with Ethics Standards All procedures followed were in accordance with the ethical standards of the responsible committee on human experimentation (institutional and national) and with the Helsinki Declaration of 1964, as revised in 2013.

Informed consent Informed assent and consent was obtained from all patients and parents, respectively.

Disclosures Tariq Aslam originally devised the RAFIhaler for his son. He has worked with and for commercial entities to make publically available products that evolved from concepts presented here and which are patent pending. $\mathrm{He}$ did not conduct patient testing, questionnaires or data collection. Savana Shakir and Clare Murray have nothing to disclose.

Data availability All data generated or analyzed during this study are included in this published article.

Authorship All named authors meet the International Committee of Medical Journal Editors (ICMJE) criteria for authorship for this manuscript, take responsibility for the integrity of the work as a whole, and have given final approval for the version to be published.

Open Access This article is distributed under the terms of the Creative Commons Attribution-NonCommercial 4.0 International License (http://creativecommons.org/licenses/ by-nc/4.0/), which permits any noncommercial use, distribution, and reproduction in any medium, provided you give appropriate credit to the original author(s) and the source, provide a link to the Creative Commons license, and indicate if changes were made. 


\section{REFERENCES}

1. Asthma UK. Asthma facts and statistics. Available at: https://www.asthma.org.uk/about/media/facts-andstatistics/ (last accessed October 2016).

2. McNeill G, Tagiyeva N, Aucott L, Russell G, Helms PJ. Changes in the prevalence of asthma, eczema and hay fever in pre-pubertal children: a 40 -year perspective. Paediatr Perinat Epidemiol. 2009;23(6):506-12.

3. Koster ES, Wijga AH, Zuidgeest MG, et al. Patterns of asthma medication use: early asthma therapy initiation and asthma outcomes at age 8 . Pharmacoepidemiol Drug Saf. 2010;19(10):991-9.

4. Leversha AM, Campanella SG, Aickin RP, Asher MI. Costs and effectiveness of spacer versus nebuliser in young children with moderate and severe acute asthma. J Pediatr 2000;136:497-502.

5. Morton RW, Everard ML, Elphick HE. Adherence in childhood asthma: the elephant in the room. Arch Dis Child. 2014;99:949-53.
6. Erzinger S, Schueepp KG, Brooks-Wildhaber J, Devadason SG, Wildhaber JH. Facemasks and aerosol delivery in vivo. J Aerosol Med. 2007;20[Suppl 1]:S78-84.

7. Chaney GM, Clements B, Laundau L, Bulsara M, Watt P. A new asthma spacer device to improve compliance in children: a pilot study. Respirology. 2004;9:499-506.

8. Burgess SW, Sly PD, Cooper DM, Devadason SG. Novel spacer device does not improve adherence in childhood asthma. Pediatr Pulmonol. 2007;42:736-9.

9. Schultz A, Sly PD, Zhang G, Venter A, Le Souef PN, Devadason SG. Incentive device improves technique but not clinical outcome in preschool children with asthma. J Paediatr Child Health. 2012;48:52-6. 